\title{
Vigilancia tecnológica e inteligencia competitiva en el modelo empresarial del sector hotelero colombiano
}

\section{Technological surveillance and competitive intelligence in the business model of the Colombian hotel sector}

\author{
Pedro Ignacio Moya-Espinosa' \\ Fabio Fernando Moscoso-Durán ${ }^{2}$
}

Recibido: octubre 18 de 2016

Aceptado: marzo 27 de 2017

\begin{abstract}
Resumen
Este artículo destaca los conceptos de Vigilancia Tecnológica, VT, e Inteligencia Competitiva, IC; con el fin de establecer su importancia para las empresas hoteleras y el papel que desempeñan en el desarrollo económico de Colombia. Se partió de una revisión teórica-conceptual de los siguientes temas: Turismo e Industria Hotelera, Globalización, Competitividad y VT e IC. Por último, se hace una propuesta que pretende motivar la aplicación de las herramientas de VT e IT para buscar un mejor nivel de desempeño empresarial del sector hotelero colombiano.
\end{abstract}

Palabras clave: vigilancia tecnológica, inteligencia competitiva, turismo, industria hotelera.

\begin{abstract}
This article emphasizes the concepts of Technological Vigilance, TV, and Competitive Intelligence, $\mathrm{Cl}$, in order to establish their importance for hotel companies and the role they play in the economic development of Colombia. It was based on a theoretical-conceptual review of the following topics: Tourism and Hotel Industry, Globalization, Competitiveness and VT and Cl. Finally, a proposal is made that aims to motivate the application of the tools of VT and IT to look for a better level of business performance in the Colombian hotel sector.
\end{abstract}

Keywords: technology watch, competitive intelligence, tourism, hotel industry.

\footnotetext{
1 Administrador de Empresas, Magíster en Ciencias de Desarrollo Empresarial, Estudiante del Doctorado en Gestión -Universidad EAN-, Universidad Pedagógica y Tecnológica de Colombia, Duitama, Colombia. E-mail: pmoyaesp3757@universidadean.edu.co

2 Economista, Doctor en Economía Internacional, Universidad EAN. E-mail: ffmoscoso@universidadean.edu.co
} 


\section{Introducción}

La hotelería es la actividad de facilitación en el turismo que se encarga del alojamiento, de ofrecer comodidad y de hacer agradable la estadía del turista en un proceso de integración con los atractivos, recursos, instalaciones, facilidades y acceso a los mismos (Vergara-Arrieta, \& Carbal-Herrera, 2014; Acle-Mena, Villalobos-Abrego, \& Herrera-López, 2016). La hotelería ha tenido una tendencia creciente debido al incremento en los últimos años registrando un crecimiento en 50 millones en número de personas que realizan actividades turísticas, alcanzando un total de 1.184 millones de viajeros en todo el mundo, según la Organización Mundial del Turismo, OMT (2016).

En un mundo cambiante, la competencia orientada por las necesidades y las expectativas del turista, el cual presenta estándares muy altos de exigencias y conocimiento frente a lo que espera encontrar en cada destino, así como en la estrategia de preparación, presentación y oferta de los mismos, subyace la satisfacción de tales expectativas y necesidades. Es allí donde la empresa hotelera encuentra su sentido y el aporte y fortalecimiento con la ayuda de la Vigilancia Tecnológica y la Inteligencia Competitiva, como instrumentos estratégicos para ser eficiente y eficaz. En este orden de ideas, García (2009), afirma que la apuesta por la calidad y diferenciación son el eje de las estrategias de las empresas y destinos turísticos.

Sancho y García (s.f.), afirman que en el entorno actual, dinámico, cambiante y de globalización de los mercados turísticos, los destinos se ven inmersos en una vorágine competitiva de todos contra todos aunque estén en lugares distantes. Por tanto, los Destinos Turísticos se enfrentan a un proceso de difícil supervivencia, donde la estrategia competitiva marca su trayectoria. De esta forma, alcanzar óptimos niveles de competitividad se convierte en un factor estratégico para los destinos turísticos. Con la Vigilancia Tecnológica e Inteligencia Competitiva, se identifica, comprende y diseña la propuesta para anticipar las acciones futuras de la empresa hotelera, para asumir los retos de la globalización, la competitividad y las exigencias del huésped de la aldea global.

Con base en lo expuesto, se pretende resolver el siguiente interrogante: ¿Cómo las empresas hoteleras, a través de las herramientas de vigilancia tecnológica e inteligencia competitiva, pueden asumir los retos de un mundo en constante cambio? En este artículo también se busca identificar cómo los sistemas de información estratégica permiten explorar el entorno en ámbitos clave, impulsando la competitividad y la capacidad anticipatoria en los hoteles colombianos, aspecto que propende por el posicionamiento, por el desarrollo económico del sector, por asumir los retos emergentes y por proponer un enfoque que permita identificar necesidades acerca de la información y su capitalización con fines de la prospección y el mejoramiento continuo.

\section{Marco teórico y metodología}

\subsection{Fundamentos conceptuales}

\section{Hotelería}

Hotelería es el conjunto de todo establecimiento de puertas abiertas al público dedicado total o parcialmente a suministrar el servicio de alojamiento y puede integrar servicios de alimentos y bebidas, diversión, eventos, SPA, deportes y otras comodidades orientadas a huéspedes permanentes $\mathrm{O}$ transeúntes. Comprende todos aquellos establecimientos que se dedican profesional y habitualmente a proporcionar alojamiento a las personas con o sin servicios de carácter complementario.

De acuerdo con la OMT (2016) hotelería es el nombre genérico de las actividades económicas que prestan el servicio ligados al alojamiento y la alimentación en una conexión más amplia con el turismo. Las principales denominaciones que reciben los establecimientos que desarrollan el tu- 
rismo son: hoteles, hostales, paradores, pensiones, albergues, casas rurales y bodegones, entre otros.

El turismo según la OMT (2016): "comprende las actividades que realizan las personas durante sus viajes y estancias en lugares distintos al de su entorno habitual, por un período de tiempo consecutivo inferior a un año, con fines de ocio, por negocios y otros motivos". También define al visitante como "una persona que visita por no más de un año un país diferente de aquél en el cual tiene de ordinario su residencia y cuyo motivo principal para la visita es distinto del de ejercer una ocupación remunerada en el mismo país que visita", con dos categorías distintas: turista y excursionista.

Turista es aquel que permanece al menos 24 horas, pero no más de un año en el país que visita y cuyos motivos de viaje pueden clasificarse así: i) placer, distracción, vacaciones, deporte; ii) negocios, visita a amigos o parientes, misiones, reuniones, conferencias, salud, estudios, religión. Además está el excursionista, también conocido como visitante del día; es aquella persona que visita un destino, pero que no pernocta en él; por tanto se desplaza de su entorno habitual con motivos distintos a ser empleado.

\section{Turismo y globalización}

El turismo es una de las actividades económicas más importantes para el desarrollo de los países; es un motor indiscutible del crecimiento económico, sobre todo, una fuente importante de empleo con amplio impacto en las comunidades locales (OMT, 2016). En este sentido, el turismo puede y debe contribuir a mejorar la calidad de vida de la población más pobre (Del Río-Cortina, 2012; Garrido-Hurtado, \& Valderrama-Cardona, 2016; Pitre-Redondo, Cardona-Arbeláez, \& Hernández-Palma, 2017).

La globalización ha llevado a todos los países a la búsqueda de herramientas necesarias para permanecer en un mercado mundial en el que so- breviven los competidores más fuertes; en este escenario los menos preparados deben diseñar mecanismos y estrategias para abrirse a nuevos mercados. En este sentido el sector turístico en particular enfrenta varios paradigmas enmarcados en las necesidades de los turistas alrededor del mundo y en las estrategias de competitividad turística que han contribuido a resaltar el papel que tienen los destinos, los centros de atracción, los complejos, los municipios turísticos y las regiones que están tomando más relevancia que los propios países a la hora de decidir un viaje. La apuesta por la calidad y la diferenciación se ha convertido en el eje de dichas estrategias en las empresas y destinos turísticos (García, 2009).

El Reporte de Competitividad de Viajes y Turismo, Foro Económico Mundial (2015), informa que Colombia ocupa el puesto 68 en el mundo, resultado que refleja cómo los bienes naturales y culturales son los fuertes que identifican al país. Sin embargo, no todo es bueno, el país no es muy competitivo en turismo, por tal motivo surge la necesidad de crear planes de mejoramiento en cuestión de calidad para así poder atraer más extranjeros al país. La competitividad de un país, especialmente en el ámbito turístico, se debe desarrollar mediante planes de calidad donde las empresas turísticas tengan la capacidad de crear nuevos planes o paquetes turísticos innovadores, y para ello es indispensable que los actores turísticos estén en un proceso de mejora continua.

Flores-Ruiz (2007), plantea que: "Muchos de los destinos turísticos tradicionales se están convirtiendo en destinos maduros, los cuales han sido visitados varias veces por un mismo turista, cada vez más experimentado, que siente la necesidad de viajar a otros lugares y vivir nuevas experiencias, pues no olvidemos que ésta es una de las motivaciones fundamentales que mueve al turista a visitar nuevos territorios". Es preciso destacar que el crecimiento de los destinos turísticos ha generado crecimiento en la competitividad, y ha generado una competencia más potente que va de la mano de la demanda turística, con el beneficio de 
que hay más utilidades en los productos que se están ofreciendo.

\section{Vigilancia tecnológica e inteligencia competitiva}

La Vigilancia Tecnológica, VT, y la Inteligencia Competitiva, IC, son dos herramientas complementarias y útiles para anticipar un suceso y mejorar la competitividad de una organización hotelera o cualquier empresa independientemente del sector al que esté circunscrita. De acuerdo con Salmi (2009), citando a La Sociedad de Profesionales de Inteligencia Competitiva, SCIP por sus siglas en inglés, la IC es un proceso ético y sistemático de recolección de información, análisis y diseminación pertinente, precisa, específica y oportuna.

La norma española sobre gestión de Investigación, Desarrollo e Innovación, I+D+l, (UNE, 2011), define Vigilancia Tecnológica como un proceso organizado, selectivo y permanente, de captar información del exterior y de la propia organización sobre ciencia y tecnología, seleccionarla, analizarla, difundirla y comunicarla, para convertirla en conocimiento para tomar decisiones con menor riesgo y poder anticiparse a los cambios. Igualmente, Concibe Inteligencia Competitiva como un conjunto de acciones coordinadas de búsqueda, tratamiento (filtrado, clasificación, análisis), distribución, comprensión, explotación y protección de la información obtenida de modo legal, útil para los actores económicos de una organización que permite el desarrollo de sus estrategias individuales y colectivas.

Autores como Morcillo (1997), afirman que inicialmente el término "vigilancia" se aplicó a la tecnología y era parte integrante de los modelos de gestión de la tecnología e innovación. Morín (1985) y Morín y Seurat (1989) proponían la vigilancia tecnológica como una función que consistía en analizar el comportamiento innovador de los competidores directos e indirectos; en explorar todas las fuentes de información (libros, literatura gris, oficinas de patentes, etc.); en examinar los productos existentes en el mercado (análisis de la tecnología incorporada) y en asistir a ferias, congresos para posicionarse respecto de los competidores y tomar así conocimiento de las tecnologías que predominarán en el futuro.

Gibbons y Prescott (1996) definen a la inteligencia competitiva como un proceso en que se obtiene, se analiza, interpreta y finalmente se divulga determinada información adquiriendo un valor estratégico sobre los competidores y sobre la industria en general; dicha información se lleva en el momento oportuno hasta los entes responsables de la toma de decisiones.

Esnal (2009), considera que una actividad de inteligencia competitiva está bien realizada si se tiene la habilidad para llegar de manera legal a las fuentes que ofrecen información útil y que permite darle respuesta a la pregunta o problema que se tiene. Igualmente, Salmi (2009) indica que IC es una acción que pretende definir, recopilar, analizar y distribuir información externa sobre productos, servicios, clientes, competidores o cualquier otro aspecto que ayuda a la toma de decisiones de la organización y al diseño de planes estratégicos y operativos. Lesca (1994) expresa que:"La vigilancia tecnológica incluye los esfuerzos que la empresa dedica, los medios de que se dota y las disposiciones que toma con el objetivo de conocer todas las evoluciones y novedades que se producen en los dominios de las técnicas que le conciernen actualmente o son susceptibles de afectarle en el futuro".

Sánchez (2008), citando a Palop (2002), propone que tanto VT e IC son definiciones que contienen expresiones que se convierten en claves para la identificación de información, tecnología y toma de decisiones; actúan como un proceso sistémico que también es reconocido como el ciclo de VT e IC, con las siguientes etapas:

i) Planeación: Es el punto de partida donde se encuentra una necesidad o una idea dentro de la organización. 
ii) Búsqueda y captación: Hay una recolección intensiva de datos, en la cual se busca, detecta y observa.

iii) Análisis y Organización: se pasa por un filtro la información donde se analiza la calidad y el tipo de información obtenida.

iv) Inteligencia: Se le da un valor a la información obtenida, para luego buscar que esta incida en la estrategia organizacional.

v) Comunicación de los resultados: En esta etapa se difunde la información a los directivos y a las áreas implicadas, para difundir y transferir el conocimiento

Hidalgo, León y Pavón (2002), plantean la diferencia que existe entre VT e IC así: "La inteligencia se diferencia de la vigilancia en que constituye un paso más en el proceso de gestión de la información obtenida. La vigilancia persigue la obtención de la información más relevante del entorno y su análisis para los intereses de la empresa; mientras que la inteligencia hace especial énfasis en otros aspectos como su presentación en un formato adecuado para la toma de decisión y el análisis de la evaluación de los resultados obtenidos mediante su uso".

La VT e IC son dos herramientas que se han convertido en fundamentales para las organizaciones que tienen procesos de $\mathrm{I}+\mathrm{D}+\mathrm{i}$, pues permiten generar nuevos proyectos a la vez que disminuyen los riesgos que puedan ser ocasionados por las actividades de esta área (Muñoz, Marín, \& Vallejo, 2006).

Con base en las fuerzas competitivas de Porter, Escorsa y Maspons (2001), resaltan que para organizar la vigilancia de una empresa se deben tener en cuenta 4 ejes:
Vigilancia Competitiva: se encarga de buscar información sobre la competencia actual y la competencia potencial.

Vigilancia Comercial: busca información de los clientes y los proveedores.

Vigilancia Tecnológica: Busca información de las tecnologías que se encuentran disponibles o que recién aparecen.

Vigilancia del entorno: busca información de hechos exteriores que puedan afectar el futuro.

Porter (1980), desde comienzos de los ochenta ya señalaba la importancia de un análisis profundo de la competencia en el diseño de la estrategia de la empresa, recomendando el empleo de sistemas formalizados de inteligencia. Y ello, precisamente por las insuficiencias que presentaban los enfoques informales. Desde entonces, la creciente adopción de enfoques formales de vigilancia e inteligencia como modo de mejorar la captación, análisis y utilización de la información ha venido siendo detectada y analizada en empresas de USA, Europa y Extremo Oriente. A partir del modelo de Porter de las fuerzas que caracterizan la posición competitiva de la empresa, Martinet y Ribault (1989) hablan de cuatro tipos de vigilancia: i) tecnológica o centrada en el seguimiento de los avances del estado de la técnica y en particular de la tecnología y de las oportunidades / amenazas que genera; ii) competitiva, implica un análisis y seguimiento de los competidores actuales, potenciales y de aquellos con producto substitutivo; iii) comercial, dedica la atención sobre los clientes y proveedores; y iv) entorno, centra la observación sobre el conjunto de aspectos sociales, legales, medioambientales, culturales, que configuran el marco de la competencia.

\subsection{Metodología}

Este trabajo se soporta en una investigación exploratoria que pretende dar una visión general de 
tipo aproximativo, respecto a la vigilancia tecnológica y a la inteligencia competitiva para la empresa hotelera de Colombia. El enfoque es cualitativo y los métodos son el deductivo que parte de lo general, de los aspectos teóricos y conceptuales y la realidad del sector empresarial objeto de estudio; y el método inductivo por medio del razonamiento lógico que permiten la aplicación de los aspectos teóricos, conceptuales en la generación de un constructo materializado en una propuesta.

\section{Propuesta de vigilancia tecnológica e inte- ligencia competitiva para el sector hotelero}

\subsection{Definición de la propuesta}

La VT y la IC son un conjunto de acciones encaminadas a la identificación, obtención, proceso, procesamiento, análisis, interpretación para la toma de decisiones y la proyección estratégica sobre la industria hotelera y sus competidores. De acuerdo con Escorsa y Maspons (2001) los japoneses para realizar un nuevo producto o iniciar una investigación, empiezan con la fase de vigilancia tecnológica y comercial, después se apropian de las tecnologías de las empresas del país, mejoran el producto, crean uno nuevo y lo comercializan a los mercados mundiales. Por lo tanto, antes de ejecutar cualquier desarrollo, tienen presente que primero hay que averiguar lo que ya se ha hecho. Japón es el país líder en VT ya que se destaca por destinar muchos fondos a la obtención de información científica y técnica.

La propuesta aquí expuesta contempla que el flujo de información entre un hotel y su entorno social, ambiental, individual y tecnológico que adquiere cada vez más importancia, siendo fundamental en la detección de oportunidades y amenazas para asumir de manera exitosa el reto competitivo. En este orden de ideas la comunicación entre la organización hotelera y su entorno tanto micro como macro ofrece un panorama de posibilidades para generar, desarrollar y asimilar nuevas capacidades tecnológicas que optimizan: tiempo, dinero y recursos, pero sobre todo busca la satisfacción del huésped, turista y en términos generales del consumidor de un mundo moderno y cada vez más exigente.

\subsection{Utilidad de la vigilancia tecnológica}

La Vigilancia Tecnológica, como proceso de actividades encaminadas a obtener información útil para la proyección de la empresa, presenta tres fases donde se destaca tal utilidad, así:

Etapa 1. Identificar información. Se identifica información clave mediante un software (captura de información) a partir de fuentes primarias o secundarias donde se destacan bases de datos, web, clientes, intermediarios, competencia, tendencias, gustos, preferencias del cliente.

Etapa 2. Proceso y procesamiento, análisis e interpretación de la información (geo ubicación, mapeo, georreferenciación, etc.). Con el apoyo de herramientas, soluciones informáticas, lenguajes y demás que permitan la sistematización, como data mining: proceso de extracción de información más útil y significativa de grandes bases de datos, información que refleja inteligencia del sector, o negocio a través de factores ocultos, tendencias, asociación, relación y correlaciones de variables clave entre otros aspectos. En turismo los Sistemas de Distribución Global, GDS por sus siglas en inglés, son intermediarios entre las compañías aéreas, las agencias de viajes y los turistas o clientes; como sistema de información de reservas abarca varias áreas del sector turístico como tiquetes o boletos aéreos, hoteles, renta de autos entre otros; se distinguen: Travel Port, Galileo, Amadeus y Sabre.

Etapa 3. Decisión estratégica. A partir de la información se puede decidir, predecir, proyectar y prospectar la organización hotelera, véase Figura 1. 


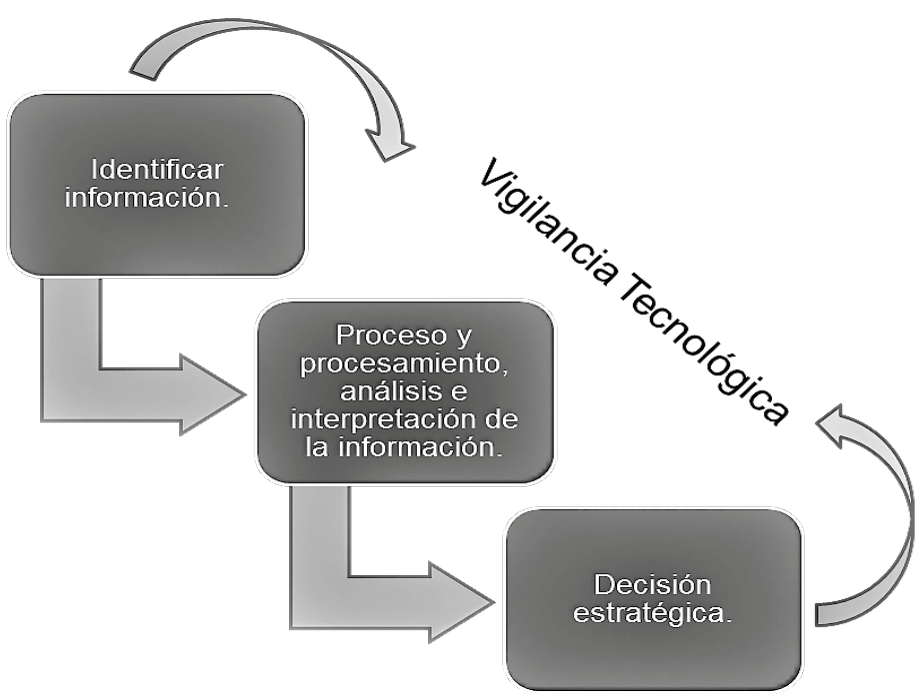

Figura 1. Utilidad de la vigilancia tecnológica para la organización hotelera.

La figura 1 muestra la utilidad que tiene la vigilancia tecnológica para la empresa, destacando en su etapa 1. La identificación de información necesaria y significativa útil para el hotel. En esta etapa se debe reconocer el mercado en el que se opera: (competencia, contexto, intereses, tendencias...), las características de los huéspedes, usuarios, turistas sus expectativas, necesidades y requerimientos, con el fin de obtener la mayor información de ellos, consolidando la información de gran interés, útil en la etapa de la decisión estratégica.

En la etapa 2. Proceso y procesamiento, análisis e interpretación, juega un papel decisivo la información de la etapa 1, con la cual se generan los constructos, esquemas y propuestas para la toma de decisiones y la proyección estratégica.

En la etapa 3. Se generan estrategias competitivas con las cuales se puede no solo reaccionar, sino adelantar a los acontecimientos, tendencias y requerimientos del entorno, es decir, el mercado, la tecnología y los clientes con todo lo que cada una de estas variables implica. Para lo anterior es preciso anotar lo expuesto por Moscoso et al (2011) que citando Krugman (2007) afirma que hace ya un tiempo advirtió que son las empresas y no los países las que compiten entre sí, estaba implícitamente definiendo la competitividad en términos de la calidad de inversión y de aumento de la productividad en un medio de estabilidad macroeconómica y de integración a la economía internacional.

3.3 Vigilancia tecnológica para los hoteles colombianos

La propuesta de la figura 2 permite resaltar que para la aplicación de una estrategia de VT e IC, es necesario concebir al hotel desde un enfoque micro y macro que parte del diagnóstico, la organización y sus características. Una vez se identifica la situación actual es conveniente contar con el juicio de expertos, para integrar referentes y posturas que pueden ser de gran ayuda en el diseño de estrategias. La obtención de la mayor cantidad de datos, procesos de información, comunicación, preferencias del huésped, desempeño de oferta, demanda, precios y valores agregados son aspectos que configuran elementos de la Vigilancia Tecnológica, Vigilancia Comercial y la Inteligencia de la competencia para generar la estrategia competitiva de la empresa hotelera. 


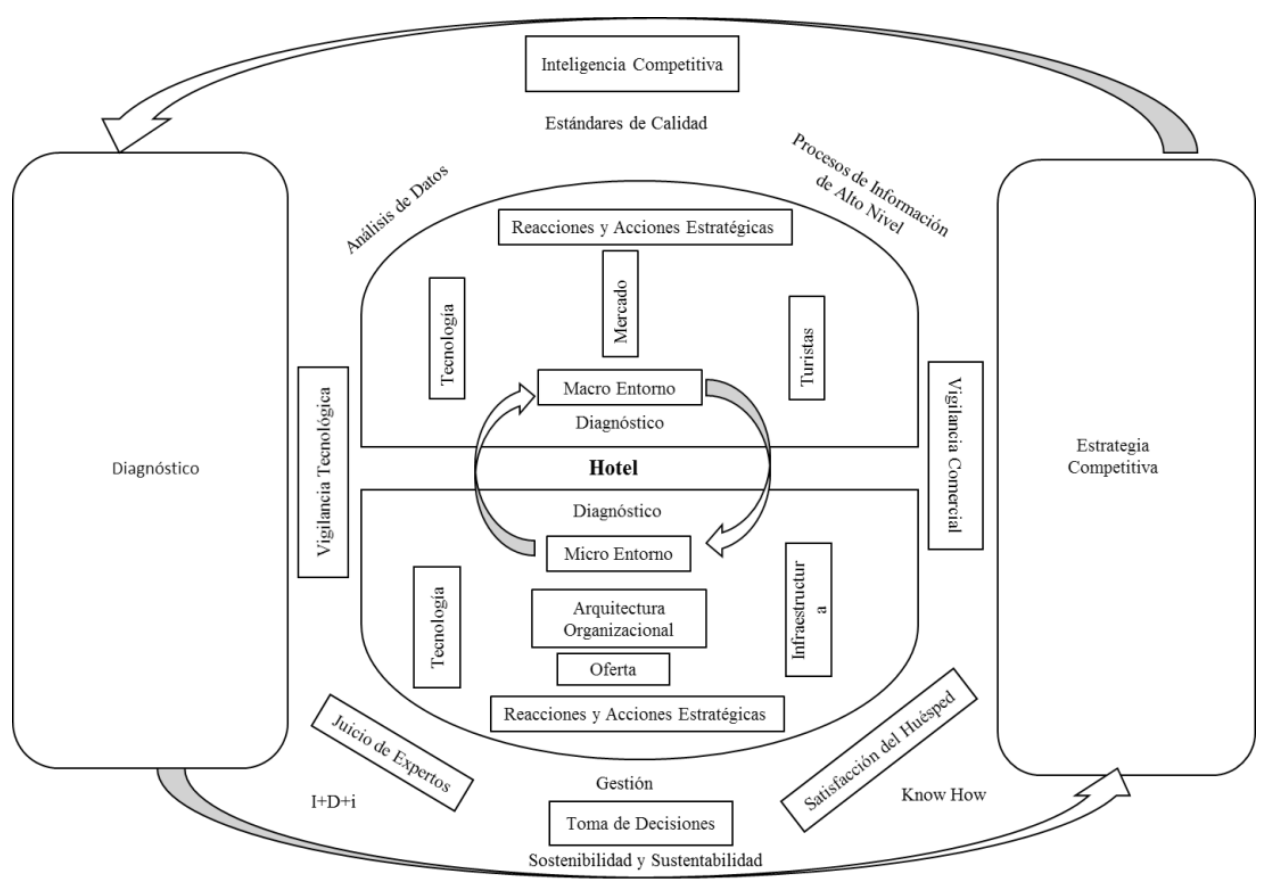

Figura 2. Propuesta de vigilancia tecnológica para los hoteles de Colombia.

En la figura 2 el diagnóstico del hotel permite la identificación de información que se puede capturar y almacenar en la estrategia de Vigilancia Tecnológica, la cual con el uso de las TIC, el trabajo colaborativo, la integración de equipos de alto desempeño, los equipos virtuosos pueden transformar los datos en información clave (Data Plannig, Data Marketing, Marketing Analitic...) y convertirla en resultados de $\mathrm{I}+\mathrm{D}+\mathrm{i}$ que se convierte en know how.

En el diagnóstico interno del hotel se identifican cómo está la estructura orgánica, los manuales de funciones, la planta de personal, los procedimientos, el talento humano (capacitación y actualización), las funciones, los procedimientos, el equipamiento, las normas de calidad y la innovación, es decir, constituir la Arquitectura organizacional y sus conexos. También es importante identificar acerca del portafolio de productos y servicios y su desempeño en cuanto a cantidad, calidad y consistencia (si cumple con lo que propone) y el grado de satisfacción del consumidor de los mismos.
El Micro entorno. Lo constituyen todos los aspectos propios del hotel y su equipamiento, un análisis frente a la estructura, infraestructura y superestructura, aunque en este análisis algunas variables son externas al hotel, están directamente relacionadas con su desempeño; por eso es conveniente conocer el estado actual que permita asumir los derroteros pertinentes en términos de competitividad a partir de la VT e IC. Por infraestructura se comprenden todos los servicios o elementos básicos que se prestan dentro del destino turístico tanto para locales como foráneos, por ejemplo vías de acceso, medios de transporte, hospitales, servicios públicos etc. La Estructura está relacionada al hotel como tal, es decir, objetos, muebles, edificios, locaciones con los que el hotel presta los servicios orientados al turista, al huésped. Ejemplo de ello, son: los hoteles, los centros recreativos, museos, oficinas de información turística, etc. La Superestructura la integran entidades privadas, públicas y de naturaleza mixta encargadas de velar e impulsar el desarrollo positivo y prospectivo del sector turístico, ejemplo de ello son: Viceministerio de Turismo y las Secretarías de Turismo municipales y 
departamentales y entidades privadas como ANATO y COTELCO, entre otras.

En cuanto al Macro entorno. Es conveniente hacer una muy buena indagación acerca de tres aspectos fundamentales: La Tecnología, el Mercado y el Turista. Tecnología, en este sentido con la VT e IC se conoce e identifica la tecnología y en conocimiento técnico de punta que permite conocer software, hardware y sus bondades en el sector hotelero. En cuanto al mercado, es importante identificar el comportamiento de la competencia, la oferta y la demanda en la región, en Colombia y en los casos exitosos del mundo, que consoliden un referente necesario e indispensable para el desarrollo de estrategias exitosas. El mercado, según Moya-Espinosa (2015), es el acuerdo mutuo de las transacciones entre individuos e instituciones, que configura el mapa estratégico de la oferta, demanda, precios, medios publicitarios, promociones e intermediación de los servicios líderes que están marcando pauta. El mercado ofrece un panorama muy amplio de opciones acerca de los productos y servicios actuales de la aldea global. El turista, es el protagonista fundamental ya que facilita conocer quién es, cómo son y cuáles son las preferencias, inclinaciones, expectativas, requerimientos y tendencias. Los gustos del consumidor, del cliente o huésped cada vez son más exigentes y porque no decirlo extraños como producto de la configuración de un turista del mundo moderno.

El modelo propuesto enfatiza en cómo la vigilancia tecnológica y la inteligencia competitiva, de manera armoniosa, configuran un proceso que le permite a la organización hotelera perdurar con éxito en el mercado dando a la misma un carácter más activo, información más elaborada; integra resultados en las diferentes necesidades: tecnológicas, mercados y turistas.

En lo relacionado con la sostenibilidad y sustentabilidad se parte de que independientemente de la categoría, clasificación, ubicación y tipo de operación de los hoteles, estos deben tener como fundamento de su diseño y gestión los principios medioambientales, sociales, culturales y económicos. Además, sus objetivos se deben direccionar a la satisfacción de necesidades de sus agentes de interés, stakeholders con profundo respeto por el entorno, la biodiversidad en el marco de la ética permanente que contribuya al crecimiento y desarrollo humano sostenible: asegurar necesidades presentes sin comprometer las necesidades de futuras generaciones, en búsqueda de un mejor capital social, calidad presente y futura. En este orden de ideas es preciso retomar a Herrera-López, Acle-Mena y Paredes-Solorio (2012), citando a la ONU (1987): algunos organismos plantean una diferencia entre el concepto de desarrollo sustentable con el de desarrollo sostenible, ya que la sustentabilidad además de cumplir con beneficios sociales, ambientales y económicos, se debe mantener en el tiempo para que pueda satisfacer las necesidades del presente sin poner en peligro la capacidad de las generaciones futuras para atender sus propias necesidades.

Igualmente, Espinoza (2008) citado por Álvarez-Meneses (2013), indica que la sostenibilidad debe ser ambiental, cultural y social, y económica, así: Sostenibilidad ambiental garantiza que el desarrollo sea compatible con el mantenimiento de los procesos ecológicos esenciales, de la diversidad biológica y los recursos. La sostenibilidad social y cultural debe garantizar que el desarrollo sostenible aumente el control de los individuos y sus vidas, y que sea compatible con la cultura y los valores de las personas y refuerce la identidad de las comunidades. Y Sostenibilidad Económica, debe garantizar que el desarrollo sea económicamente eficiente, beneficie a toda la gente de la región afectada y que los recursos sean gestionados de manera que se conserve para las generaciones futuras.

Una vez se aborda el diagnóstico, se pasa a la consolidación de la estrategia competitiva que a su vez se traduce en beneficios para la organización, para el huésped y para la sociedad en general; y todo el modelo dimensionado con criterios de sostenibilidad y sustentabilidad para garantizar 
la permanencia en el tiempo con responsabilidad ambiental, económica, social y cultural.

\section{Conclusiones}

El ejercicio de la vigilancia tecnológica se encuentra más directamente vinculado con la captación y análisis intrínsecos de las informaciones, mientras que el desarrollo de la inteligencia competitiva está orientada hacia la interpretación de esas informaciones previamente seleccionadas para ayudar a la toma de decisiones.

La Vigilancia tecnológica e Inteligencia Competitiva configuran un proceso de identificación, monitoreo, hallazgos, obtención, análisis, interpretación y difusión de información de valor estratégico sobre el hotel, el sector y los competidores, útil para la toma de decisiones en el momento oportuno que permite anticipar la acción.

El turismo actual se caracteriza por la existencia de turistas con más conocimiento e información y con destinos maduros, donde los hoteles como facilitadores de tal actividad precisan de un sistema de Inteligencia Competitiva que les ayude y oriente en la consecución y análisis de información útil para afrontar con éxito el monitoreo de los servicios actuales y los nuevos; sobre la evolución global de los hoteles referentes y competidores no solo en sus portafolios, sino en facturación y demás temas de prospección a partir de datos en tiempo real.

Un sistema de VT e IC para un hotel es de gran ayuda debido a la evolución de los requerimientos del mercado, de zonas geográficas, oferta de servicios a partir de la identificación y consulta de sistemas rigurosos de fuentes de información adecuada para el sector; esto se traduce en oportunidades en el contexto competitivo ya que permite la detección de señales de cambio, análisis, tendencias, reacción, estrategia de la competencia, así como nuevas amenazas y oportunidades.

\section{Agradecimientos}

Los autores agradecen a Colciencias y a Colfuturo por el financiamiento de la investigación que da origen a este artículo, como parte de la convocatoria regiones 733 de 2015.

\section{Referencias}

Acle-Mena, R. S., Villalobos-Abrego, C., \& Herrera-López, B. (2016). Reposicionamiento del Barrio del Artista para aumentar su valor cultural-artístico en Puebla, México. Revista de Investigación, Desarrollo e Innovación, 6 (2), 109-116. Doi: http://doi. org/10.19053/20278306.4601

Alvarez-Meneses, T. (2013). La planificación turística: un aspecto clave para el desarrollo sostenible y regional de Boyacá. Revista de Investigación, Desarrollo e Innovación, 3 (2), 101-110. doi:http://dx.doi. org/10.19053/20278306.2169

Del Río-Cortina, J. (2012). Desarrollo de actividades de emprendimiento con innovación y responsabilidad social en los hoteles en la ciudad de Cartagena de Indias. Saber, Ciencia y Libertad, 7 (2), 113-124. Recuperado de: https://dialnet.unirioja. es/servlet/articulo?codigo $=5109395$

Escorsa, P., \& Maspons, R. (2001). De la vigilancia tecnológica a la inteligencia competitiva. Madrid, España: Editorial Financial Times Prentice Hall.

Esnal, L. (2009) Inteligencia de mercado en Latinoamérica. CNN. Recuperado de: http://www.cnnexpansion.com.

Flores-Ruiz, D. (2007). Competitividad sostenible de los espacios naturales protegidos como destinos turísticos: un análisis comparativo de los parques naturales Sierra de Aracena y Picos de Aroche y Sierras de Cazorla, Segura y las Villas (Tesis doctoral). Universidad de Huelva. España. 
Foro Económico Mundial (2015) Reporte de competitividad en viajes y turismo. Recuperado de: http:// imco.org.mx/competitividad/reporte-de-competitividad-en-viajes-y-turismo-2015-via-wef/

García, G. (2009). El concepto de competitividad sistémica. Revista Universidad de Sonora, 23, 01-63. Recuperado de: http--www.revistauniversidad. uson.mx-revistas-25-8.pdf

Garrido-Hurtado, D., \& Valderrama-Cardona, E. (2016). Identificación de las ideas de negocio para la creación de empresa en la población desplazada de Florencia, Colombia. Revista de Investigación, Desarrollo e Innovación, 7 (1), 37-48. doi:https:// doi.org/10.19053/20278306.v7.n1.2016.5634

Gibbons, P., \& Prescott, J. (1996). Parallel Competitive intelligence processes in organizations. International Journal of Tecnology Management, 11 (12), 162-178.

Herrera-López, B., Acle-Mena, R., \& Paredes-Solorio, R. (2012). Evaluación de recursos para el aprovechamiento del turismo de naturaleza en el Área Natural Protegida Estatal "Sierra del Tentzo" Puebla, México. Revista de Investigación, DesarroIlo e Innovación, 3 (1), 8-17. Recuperado de http:// revistas.uptc.edu.co/revistas/index.php/investigacion_duitama/article/view/2132

Hidalgo, A., León, G., \& Pavón, J. (2002). La gestión de la innovación y la tecnología en las organizaciones. Madrid, España: Editorial Pirámide.

Lesca, H. (1994) Veille stratégique, l'intelligence de I'entreprise. Aster, Gières. Recuperado de: https:// www.madrimasd.org/revista/revista17/tribuna/ tribuna1.asp.

Martinet, B., \& Ribault, B. (1989). La Veille Technologique, Concurrentielle et Commerciale: Sources, Methodologie, Organisation. París, Francia: Les Editions d'Organisation.
Morcillo, P. (1997). La dirección estratégica de la tecnología e innovación. Madrid, España: Civitas.

Morín, J. (1985). L'excellence technologique. París, Francia: Publi Union.

Morín, J., \& Seurat, R. (1989). Le management des ressources technologiques. París, Francia: Les Editions d'Organisation.

Moscoso, F., et al. (2011) Informe Anual de Competitividad de Cundinamarca 2010-2011. Recuperado de: http://www.cundinamarca.gov.co/wps/wcm/connect/adb303c3-e057-4741-a2f7-caa569d05918/ INF+COMPETITIV+CUND+22011+AJUSTADO.pd$\mathrm{f}$ ?MOD=AJPERES.

Moya, P. (2015). Introducción a la Mercadotecnia. Un acercamiento a la satisfacción de necesidades. Tunja, Colombia: Editorial UPTC.

Muñoz, J., Marín, M., \& Vallejo, J. (2006). La vigilancia tecnológica en la gestión de proyectos de I+D+i: recursos y herramientas. Revista El Profesional de la Información, 15 (6), 411-419. Recuperado de: http://atarazanas.sci.uma.es/docs/articulos/16600770.pdf

OMT (2016) Datos Organización Mundial del Turismo. Recuperado de: http://media.unwto.org/ es/press-release/2016-01-18/las-Ilegadas-de-turistas-internacionales-suben-en-2015-un-4-hasta-el-record file:///C:/Users/familia/Downloads/ OEE_DO_WA_Turismo_Mayo_01-07-2016.pdf

Pitre-Redondo, R., Cardona-Arbeláez, D., \& Hernández-Palma, H. (2017). Proyección del emprendimiento indígena como mecanismo de competitividad en el postconflicto colombiano. Revista de Investigación, Desarrollo e Innovación, 7(2), 231240. doi:https://doi.org/10.19053/20278306. v7.n2.2017.6068 
Porter, M. (1980). Estrategia Competitiva. Técnicas Para el Análisis de los Sectores Industriales y de las Competencias. México: CECSA.

Salmi, J. (2009). The challenge of establishing World-Class Universties. Washington, USA: The world bank. Recuperado de: http://www.scip.org/ content.cfm?itemnumber $=2214 \&$ navltemNumber $=492$

Sánchez, J. (2008). Apoyo en la definición de políticas públicas en ciencia, tecnología e innovación a través de vigilancia tecnológica e inteligencia competitiva. En: Estudios de vigilancia tecnológica aplicados a cadenas productivas del sector agropecuario colombiano. Bogotá, Colombia.

Sancho, A. \& García, G. (s.f.). El posicionamiento de un destino turístico en un mercado globalizado y competitivo: comparación de prácticas entre competidores líderes. Recuperado de: htpp://iei.uv.es/ docs/ponencias/posicionamiento_turístico.pdf.

UNE 166006:2011 (2011). Norma Española sobre Gestión de la l+D+i Sistemas de Vigilancia Tecnológica e inteligencia competitiva. Madrid, España. Recuperado de: https://www.isotools.org/2012/02/14/ une-1660062011-ex-gestion-de-la-idi-sistema-de-vigilancia-tecnologica-e-inteligencia-competitiva/

Vergara-Arrieta, J., \& Carbal-Herrera, A. (2014). Diseño de un sistema de gestión en responsabilidad social empresarial para pequeños hoteles en la ciudad de Cartagena. Saber, Ciencia y Libertad, 9 (2), 91-108. doi:http://dx.doi.org/10.22525/sabcliber.2014v9n2.91108 